\title{
Harnessing anti-idiotypic antibody alternatives to advance biotherapeutic pharmacokinetic assays
}

\author{
Matt Johnson*,1 \\ ${ }^{1}$ Avacta Life Sciences, Ash Way, Thorp Arch Estate, Wetherby, LS23 7FA, UK \\ *Author for correspondence: affimers@avacta.com
}
'Galternative binding reagents are increasingly being explored that could supplement, or in some cases supersede, antibodies in PK assays"

First draft submitted: 2 December 2019; Accepted for publication: 2 December 2019; Published online: 24 January 2020

Keywords: affimer $\bullet$ antibody $\bullet$ assay development $\bullet$ binding reagents $\bullet$ biotherapeutics drug development $\bullet$ ELISA - pharmacokinetic assays $\bullet$ therapeutics

Pharmacokinetics (PK) is the branch of pharmacology dedicated to determining the fate of substances in the body. PK assays are, therefore, an essential element in the development of new drugs, with favorable PK profiles essential to promising leads progressing to the later stages of development. In combination with pharmacodynamic (PD) assays, PK assays are vital to understanding a drug's mechanism of action, and it is increasingly recommended that they be carried out throughout the drug-development process for a more efficient pipeline [1]. PK assays can also be used as companion diagnostics at the point-of-care by determining the concentration of a therapeutic in a patient's blood; it is possible to assess whether the patient is getting the right dose; and a clinician can use this information to make personalized adjustments where appropriate [2].

To be effective, PK assays must accurately measure a drug's concentration in complex biological matrices such as blood plasma. This requires a reliable supply of reagents that can bind to different drug molecules with the highest possible specificity. The most commonly used platforms for PK assays are ligand-binding assays, and in particular, enzyme-linked immunosorbent assay (ELISA) formats employing tagged antibodies to bind to the drug of interest. The validity of PK readouts is largely determined by both the assay format, and the quality of the reagents used. Although antibodies are traditionally the preferred binding reagents, questions have been raised by some about their suitability in a number of situations. This generally stems from antibodies' inherent structural complexity, which can lead to associated issues with stability, development speed, production costs, lot-to-lot variability and target specificity [3,4]. These issues can be so problematic that erroneous conclusions may be drawn from biochemical assays, leading some to blame antibodies for the 'reproducibility crisis' currently being seen across the life science research sector [4].

If the drug being measured by the PK assay is itself an antibody, then this can complicate matters even further. In such cases the PK binding reagent must be anti-idiotypic (anti-ID), which means it must bind to the unique complementarity-determining region of the therapeutic antibody in a highly selective manner. The challenge here is ensuring the PK reagent is specific to the therapeutic antibody, and exhibits minimal cross-reactivity to the excess of antibodies found in human blood plasma [5].

Anti-ID reagents bind to therapeutic antibodies in three ways:

- Competing: used to measure free drug concentration, unbound to the target;

- Noncompeting: used to measure total drug concentration (free and bound);

- Complex specific: used to measure the concentration of the drug bound to the target.

Each binding mode has a different utility across the design and development of various assay formats, depending on the therapeutic in question. Independent of its binding mode, the anti-ID reagents' quality is essential for 
successful assay development, requiring high specificity, sensitivity and reproducibility, all of which are factors that can be limited when using antibodies.

Owing to the difficulties described above, alternative binding reagents are increasingly being explored that could supplement, or in some cases supersede, antibodies in PK assays. There are a range of potential engineered 'antibody alternative' binders currently available in various formats, including nanoCLAMPs [6], anticalins [7], affibodies [8] and Affimer ${ }^{\circledR}$ binders [9]. Affimer protein scaffolds are particularly well suited for commercial anti-ID applications.

\section{Introducing Affimer technology}

Affimer proteins are based on a scaffold of naturally occurring proteins called cystatins. They are engineered to stably display two variable loops, which gives rise to a potential diversity of $4^{22}$ unique binding surfaces. At $14 \mathrm{kDa}$, they are just $1 / 10^{\text {th }}$ the size of the average antibody. The structural simplicity of Affimer molecules is key to their advantages. Expressed as a single domain, they have no disulfide bridges or post-translational modifications - this simplifies their production, minimizing the risk of structural variation and resultant loss of assay consistency, a major issue seen with antibodies used as anti-ID reagents.

In contrast to anti-ID antibodies, which typically take 6-9 months to be developed, Affimer reagents can be developed in a short timeframe of 12-14 weeks. This process can be completed without the need for the additional affinity maturation often required for antibody binders - a lengthy, iterative series of steps that can introduce instabilities into the protein structure [10]. Extensive standardization to ensure consistency between lots is often necessary when using antibodies in PK assays. However, due to the simple process required to produce Affimer binders, recombinant protein variability is significantly minimized between lots, resulting in a high level of reproducibility and lot-to-lot consistency.

\section{Demonstrating Affimer utility}

The utility of Affimer reagents for PK assays has been demonstrated by identifying specific binders to the existing therapeutic monoclonal antibody Trastuzumab, in a short timeframe. The roughly 3-month development process involves using gene libraries to encode variable loops, with Affimer proteins binding specifically to the target therapeutic antibody being rapidly selected by phage display experiments. Cloning the successful binding phage outputs into an expression vector generates a reliable supply of the desired Affimer binder. The properties of the Affimer binder can be examined by using it as a capture reagent in an IgG-detection sandwich enzyme-linked immunosorbent assay, validating its specific binding to the biotherapeutic over a clinically relevant concentration range.

Using this rapid discovery and development process, Affimer binders for a number of different therapeutic antibodies including rituximab, adalimumab, eculizumab, nivolumab, ipilimumab and pembrolizumab have been identified, and have been shown to accurately and precisely quantify the target antibody within a bioanalytical assay environment, yielding highly reproducible calibration curves [11].

Standard anti-ID antibodies or fragments can lack specificity due to some level of cross-reactivity to background human IgGs present at high concentration in serum, resulting in inaccurate analysis. Specificity of reagents for target biotherapeutic antibodies is essential to their performance within patient samples. Upon incubation with the target therapeutic antibody, Affimer reagents have been shown to maintain specificity to their target antibodies in the presence of matrix, and to exhibit minimal interaction with other antibody therapeutics [5], illustrating the highly specific nature of the binders. This is valuable in assessing PKs during clinical trials and in determining optimal dosing windows for patients, because the accuracy of drug measurement is retained despite variation in human antibody levels across different blood samples.

Different matrices will always give rise to slightly different background results, but minimizing the effect of matrix differences can drastically simplify PK assay setup, and may help move away from the need to run bridging assays. Using a generic secondary reagent, it was possible to measure the Affimer reagent capture of a therapeutic monoclonal antibody, with minimal matrix interference at different serum concentrations $[5,11]$. However, when using commercially available antigen-binding fragments as capture reagents, significant matrix effect is observed, leading to reduced dynamic range in serum [5,11]. While Affimer reagents exhibit reduced matrix interference, any issues in different sera can easily be mitigated using readily available assay optimization tools such as protein-free blockers [11].

In addition to flexibility in the choice of detection reagents, Affimer binder assay performance can also be modulated using defined surface options to tweak dynamic range or increase sensitivity, when all other assay 
conditions are fixed. This modulation is easily achieved by titrating nonbinding scaffold proteins into the assaycoating mixture to spread surface distribution of functional binding sites. Sensitivity can be further driven by making multidomain Affimer and bi-paratopic capture surfaces [11].

The supply of anti-ID reagents must be both stable and secure in order to meet quality-control requirements for critical reagents and minimize batch variation. As monoclonal antibodies are incredibly time-consuming to produce and are at high risk of batch-to-batch variability, it is necessary to maintain banks of hybridomas or cell lines for quick recovery for consistent production. This also poses a risk in case of disaster causing cell banks to be lost. Owing to the simplicity of the Affimer reagent production process, it is much cheaper and quicker to reliably remake Affimer gene constructs should these be lost. It is also possible to store Affimer reagents stably for long time periods when dried on plate surfaces, even at room temperature. This has been demonstrated using Affimer anti-ID binders for adalimumab, rituximab and trastuzumab, which showed no significant loss of assay performance after 3 months at $25^{\circ} \mathrm{C}[11]$.

\section{Conclusion}

The efficacy and reliability of drug development and diagnostics, of which outputs from PK assays are an essential part, is dependent on the quality of each individual process involved. It is, therefore, vital that researchers challenge and investigate these steps, and the reagents used. Certain limitations posed by the use of traditional antibody molecules may be overcome by high-quality alternatives, such as Affimer binders. These alternatives offer flexibility and specificity to ensure consistent and stable processes within both drug development and diagnostics, helping to speed the development of new biologic medicines, and to define more targeted dosing windows for patients.

Financial \& competing interests disclosure

Matt Johnson is Chief Technology Officer at Avacta Life Sciences, developer of Affimer ${ }^{\circledR}$ technology. Affimer proteins have applications across diagnostics, drug/biomarker discovery, biotech research and development, and therapeutics. The author has no other relevant affiliations or financial involvement with any organization or entity with a financial interest in or financial conflict with the subject matter or materials discussed in the manuscript apart from those disclosed.

No writing assistance was utilized in the production of this manuscript.

\section{References}

Papers of special note have been highlighted as: • of interest; $\bullet \bullet$ of considerable interest

1. Tuntland T, Ethell B, Kosaka T et al. Implementation of pharmacokinetic and pharmacodynamic strategies in early research phases of drug discovery and development at Novartis Institute of Biomedical Research. Front. Pharmacol. 5, 174 (2014).

- Outlining the importance of pharmacokinetic (PK) assays throughout the drug discovery and development process.

2. Lee C, Park C, Hsiao C. Abstract 4659. Point of care PK quantitation device for pharmacokinetic guided dosing of paclitaxel as a companion diagnostic device. Poster presented at: American Association for Cancer Research Annual Meeting 2014. CA, USA, 5-9 April 2014 .

- Demonstrating an application of PK assays in point-of-care companion diagnostics.

3. Paul KF, McLeod J. Restructuring proteomics: the rise of next-generation affinity reagents. Drug Discov. World Winter issue, 17-22 (2014).

4. Baker M. Reproducibility crisis: blame it on the antibodies. Nature 521(7552), 274-276 (2015).

- Outlining some of the features of antibodies that can lead to reproducibility issues, and the implications this has for the 'reproducibility crisis'.

5. Nuttall J, Nicholl A, Ford R et al. Poster POS028. Affimer ${ }^{\circledR}$ anti-idiotypic binders: high-performing critical reagents for diverse clinical needs. Poster presented at: 4th European Bioanalysis Forum Young Scientist Symposium. Bologna, Italy, 21 March 2019.

6. Suderman R, Rice D, Gibson S, Strick E, Chao D. Development of polyol-responsive antibody mimetics for single-step protein purification. Protein Expr. Purif. 134, 114-124 (2017).

7. Skerra A. Alternative binding proteins: anticalins - harnessing the structural plasticity of the lipocalin ligand pocket to engineer novel binding activities. FEBS J. 275(11), 2677-2683 (2008).

8. Surat P. Affibody Molecule Discovery and Uses (2018).

https://www.news-medical.net/life-sciences/Affibody-Molecule-Discovery-and-Uses.aspx

9. Tiede C, Bedford R, Heseltine S et al. Affimer proteins are versatile and renewable affinity reagents. eLife 6, e24903 (2017).

- Outlining a discovery process for Affimer reagents, and demonstrating their potential as alternative affinity reagents to antibodies. 
10. Julian MC, Li L, Garde S, Wilen R, Tessier PM. Efficient affinity maturation of antibody variable domains requires co-selection of compensatory mutations to maintain thermodynamic stability. Sci. Rep. 7, 45259 (2017).

- Describing the lengthy process of affinity maturation often required to develop monoclonal antibody affinity reagents.

11. Johnson M. Improving PK and drug monitoring assays with the Afiimer ${ }^{\circledR}$ Platform. Presented at: $12^{\text {th }}$ European Bioanalysis Forum Open Symposium, Barcelona, Spain, 21 November 2019.

-. Describing a series of experiments that demonstrate the utility of Affimer anti-ID reagents in PK assays, through development and optimization of Affimer binders for a number of therapeutic antibodies. 